\title{
COMMENT
}

\section{The NIH ECHO Program: investigating how early environmental influences affect child health}

\author{
Carol J. Blaisdell ${ }^{1}$, Christina Park ${ }^{1}$, Manjit Hanspal ${ }^{1}$, Mary Roary ${ }^{1}$, S. Sonia Arteaga ${ }^{1}$, Susan Laessig ${ }^{1}$, Erin Luetkemeier ${ }^{1}$, \\ Matthew W. Gillman ${ }^{1}$ and on behalf of program collaborators for Environmental influences on Child Health Outcomes
}

Pediatric Research (2022) 92:1215-1216; https://doi.org/10.1038/s41390-021-01574-8

In September 2016, the National Institutes of Health (NIH) launched a 7-year extramural research program, Environmental influences on Child Health Outcomes (ECHO; https://echo.nih.gov). The ECHO Program has two major components, pediatric cohorts -focusing on observational research-and the IDeA States Pediatric Clinical Trials Network (ISPCTN)-focusing on interventional research. The program's mission is to enhance the health of children for generations to come. ECHO's goals are to (1) enable high-impact research evidence that will inform clinical practices, policies, and programs for child health and development; and (2) establish a national data platform and biorepository for the scientific community. This article discusses the observational research component of $\mathrm{ECHO}$ and introduces a series of three reviews $^{1-3}$ appearing in this issue or forthcoming, each covering a different aspect of the ECHO Program.

ECHO supports multiple synergistic, longitudinal studies to investigate how environmental exposures in early life-including physical, chemical, social, behavioral, biological, natural, and built environments-affect child health and development. ECHO science focuses on five key pediatric outcomes that have a high public health impact: (1) pre-, peri-, and postnatal outcomes; (2) upper and lower airway conditions; (3) obesity; (4) neurodevelopment; and (5) positive health. ECHO uses a collaborative team science approach to children's health research. ${ }^{1}$

$\mathrm{NIH}$ designed the ECHO pediatric cohorts by leveraging existing longitudinal birth cohorts, and supporting expansion of recruitment and continued follow-up of pregnant women and their children. Through this new approach, NIH engaged researchers with expertise in participant engagement and innovative data and biospecimen collection. The ECHO pediatric cohorts comprise 31 $\mathrm{NIH}$ awards, supporting 69 unique birth cohorts that began enrolling over the past 25 years, many during pregnancy. Overall, the pediatric cohorts represent $>57,000$ child participants from diverse backgrounds across the United States. Cohorts are coordinated with and supported by a Coordinating Center, Data Analysis Center, Person-Reported Outcomes Core, Genetics Core, and the National Institute of Environmental Health Sciences (NIEHS) Human Health Exposure Analysis Resource (HHEAR; https://hhearprogram.org/).

Given the variety of cohort-specific protocols, ECHO researchers developed an ECHO-wide Cohort Data Collection Protocol (EWCP), ${ }^{1}$ which supports a common approach to data and biospecimen collection, for all cohorts to implement. The EWCP specifies the data elements for ongoing data collection, as well as for submitting data and biospecimens that cohorts collected before ECHO onto the ECHO-wide cohort data platform. The protocol reflects a broad array of information on early-life exposures and ECHO intermediate, and targeted child health outcomes specified by life stage (pregnancy, infancy, early childhood, middle childhood, and adolescence). The protocol strikes a balance between depth and breadth of the data needed to answer child health research questions, while considering feasibility of data collection. The EWCP and forms are publicly available for all researchers at https://www.echochildren.org.

The EWCP specifies a variety of essential and recommended biospecimens to collect at each life stage. ECHO developed biospecimen collection, processing, and storage guidance for blood, urine, stool, and placenta samples from pregnant mothers and their children. ECHO stores newly collected biospecimens in the $\mathrm{ECHO}$ biorepository managed by Fisher Bioservices. Cohorts also contribute previously collected biospecimens to ECHO-wide cohort science in two important ways: (1) by transferring existing bioassay data to the ECHO-wide cohort data platform and (2) by sending specific biospecimen samples to the HHEAR laboratories for approved ECHO-wide cohort analyses.

NIH collaborates with the NIEHS HHEAR laboratories to perform state-of-the-art exposure bioassays for targeted and untargeted analytes. In coordination with ECHO researchers, HHEAR labs developed targeted multi-chemical panels (e.g., metals, plasticizers, pesticides, perfluorinated chemicals, nutrients, and tobacco biomarkers), special biomarkers (tooth assays that reconstruct past exposures in pregnancy), ${ }^{4-7}$ and untargeted chemical assays (e.g., metabolic biomarkers). ECHO investigators will use the bioassay data to establish $\mathrm{ECHO}$-wide cohort population reference ranges, track exposure trends over time or by life stage, identify characteristics of participants with higher/lower exposures, and investigate relationships between exposures and children's health outcomes in the ECHO-wide cohort.

ECHO investigators periodically amend the EWCP to enhance and incorporate new data elements, such as those for rapid response to emerging public health needs. For example, ECHO investigators added data elements for medical record abstraction to support research on how early opioid exposure affects child health and development. ${ }^{8}$ In less than a month, ECHO developed surveys related to COVID-19 and obtained central IRB approval. Due to the interruptions in in-person visits during the COVID-19 pandemic, ECHO investigators are exploring innovative remote options to achieve ECHO goals for new data and biospecimen

\footnotetext{
${ }^{1}$ National Institutes of Health, Bethesda, MD, USA

Correspondence: Carol J. Blaisdell (carol.blaisdell@nih.gov)

See Acknowledgments for a full listing of collaborators
}

Received: 9 April 2021 Revised: 21 April 2021 Accepted: 29 April 2021

Published online: 15 June 2021 
collection. ECHO practices the team science approach to find creative ways to tackle short- and long-term child health concerns.

Since 2016, ECHO investigators have published over 600 journal articles (see https://echochildren.org/echo-program-publications/). Tylavsky et al. demonstrated with standardized data from 70 $\mathrm{ECHO}$ cohorts that this national study population of diverse child participants has the power to detect variation in body mass index prevalence by age and race/ethnicity. ${ }^{9}$ Among ECHO's publications, investigators have identified high-priority analyses needed to fill knowledge gaps for children's environmental health research. ${ }^{9-11}$

The ECHO Program supports the development of the next generation of children's health researchers by providing opportunities for diverse, motivated early scientists to conduct transformative, solution-oriented research. Since 2018, ECHO has funded the Opportunities and Infrastructure Fund (OIF) for early investigators (students, residents, research fellows, postdocs, and faculty who have not served as a program director or principal investigator on an NIH independent research award) to develop innovative projects that introduce new research, tools, and technologies into ECHO. ${ }^{2}$ In 2020, the ECHO Program offered Diversity Supplements for Graduate (Predoctoral) and Health Professional Students and Individuals in Postdoctoral Training (see https://grants.nih.gov/grants/guide/pa-files/PA-21-071.html) to build a workforce including individuals from underrepresented groups. The ECHO OIF and Diversity Supplements support ECHO scientific discoveries and innovations, encourage growth of investigators to become independent, and advance child health research that impacts programs, policies, and practices.

ECHO supports innovative approaches to program coordination. With over 1200 researchers, ECHO coordinates policies, procedures, collaborative working spaces, communications, and data analyses to enhance child health and develop a diverse workforce. $^{1-3} \mathrm{ECHO}$ will serve as a national resource with publicly available protocols for longitudinal birth cohorts, standardized data collection forms, and high-quality, harmonized data and biospecimen repositories to enable research to enhance children's health for generations to come. ${ }^{3}$

\section{ACKNOWLEDGEMENTS}

The authors wish to thank our ECHO colleagues, the medical, nursing and program staff, as well as the children and families participating in the ECHO cohorts. We also acknowledge the contribution of the following ECHO Program collaborators: Coordinating Center: Duke Clinical Research Institute, Durham, North Carolina: Smith P.B., Newby K.L., and Benjamin D.K. Research reported in this publication was supported by the Environmental influences on Child Health Outcomes (ECHO) program, Office of The Director, National Institutes of Health. The content is solely the responsibility of the authors and does not necessarily represent the official views of the National Institutes of Health.

\section{AUTHOR CONTRIBUTIONS}

All authors contributed to the conception and design of this manuscript, and participated in its drafting. All authors have provided final approval of the version submitted for publication.

\section{ADDITIONAL INFORMATION}

Competing interests: The authors declare no competing interests.

Publisher's note Springer Nature remains neutral with regard to jurisdictional claims in published maps and institutional affiliations.

\section{REFERENCES}

1. LeWinn, K. Z., Caretta, E., Davis, A., Anderson, A. L. \& Oken, E. Program Collaborators for Environmental influences on Child Health Outcomes. SPR perspectives: Launching the Environmental influences on Child Health Outcomes (ECHO) Program and overcoming challenges to generate engaged, multidisciplinary science. Pediatr. Res. (2021, this issue).

2. Romano, M. E., Buckley, J. P., Elliott, A. J., Johnson, C. C. \& Paneth, N. Program Collaborators for Environmental influences on Child Health Outcomes. SPR perspectives: scientific opportunities in the Environmental influences on Child Health Outcomes (ECHO) Program. Pediatr. Res. (2021, in press).

3. Jacobson, L., Parker, C., Cella, D., Mroczek, D. \& Lester, B. Program Collaborators for Environmental influences on Child Health Outcomes. SPR Perspectives: Approaches to protocol standardization and data harmonization in the ECHOwide Cohort Study. Pediatr. Res. (2021, manuscript in preparation).

4. Arora, M. et al. Determining prenatal, early childhood and cumulative long-term lead exposure using micro-spatial deciduous dentine levels. PLoS ONE 9, e97805 (2014).

5. Arora, M. \& Austin, C. Teeth as a biomarker of past chemical exposure. Curr. Opin. Pediatr. 25, 261-267 (2013).

6. de Water, E. et al. Early-life dentine manganese concentrations and intrinsic functional brain connectivity in adolescents: a pilot study. PLOS ONE 14, e0220790 (2019).

7. Padula, A. M. et al. A review of maternal prenatal exposures to environmental chemicals and psychosocial stressors-implications for research on perinatal outcomes in the ECHO program. J. Perinatol. 40, 10-24 (2020).

8. Conradt, E. et al. Prenatal opioid exposure: neurodevelopmental consequences and future research priorities. Pediatrics 144, e20190128 (2019).

9. Tylavsky, F. A. et al. Understanding childhood obesity in the US: the NIH environmental influences on child health outcomes (ECHO) program. Int. J. Obes. 44, 617-627 (2020)

10. Pellizzari, E. D. et al. Identifying and prioritizing chemicals with uncertain burden of exposure: opportunities for biomonitoring and health-related research. Environ. Health Perspect. 127, 126001 (2019).

11. Buckley, J. P. et al. Opportunities for evaluating chemical exposures and child health in the United States: the Environmental influences on Child Health Outcomes (ECHO) Program. J. Expo. Sci. Environ. Epidemiol. 30, 397-419 (2020). 